\title{
ESTUDO COMPARATIVO DE MÉTODO DIFERENCIAL TERMOFLUIDODINÂMICO PARA TROCADORES DE CALOR DO TIPO CASCO E TUBOS 1-2 COM CHICANAS FRACIONADAS E HELICOIDAIS
}

\author{
A. S. PEREIRA ${ }^{1}$, M. L. MAGALHÃES ${ }^{1}$ e S. J. M. CARTAXO ${ }^{1}$ \\ ${ }^{1}$ Universidade Federal do Ceará, Departamento de Engenharia Química \\ E-mail para contato: samuel@ufc.br
}

\begin{abstract}
RESUMO - Trocadores de calor do tipo casco e tubos são equipamentos utilizados para a manipulação e reaproveitamento de cargas térmicas entre correntes de processo. Neste trabalho, utilizou-se do desenvolvimento de um método rigoroso transiente de análise e dimensionamento de trocadores de calor casco e tubos 1-2 para comparação do comportamento termofluidodinâmico como coeficientes de transferência de calor e perda de carga para diferentes conformações de chicanas: fracionadas e helicoidais. Para isso, os parâmetros do trocador foram mantidos constantes, com o objetivo de avaliar o desempenho em diferentes condições operacionais. Foram utilizados os métodos apresentados por Bell-Delaware (chicanas fracionadas) e Tao et al. (chicanas helicoidais) para o lado do casco e as propriedades físicas são avaliadas localmente baseadas em equações de predição adaptáveis a vários fluidos disponíveis em banco de dados e na literatura. As simulações foram realizadas no ambiente computacional framework Python e seus módulos, para determinar o perfil de temperatura, pressão e propriedades físicas dos fluidos ao longo do trocador, considerando a variação unidimensional. Esta ferramenta é de grande ajuda na análise e dimensionamento de trocadores de calor, pois podemos determinar qual o tipo de arranjo de chicana é mais adequado para cada processo.
\end{abstract}

\section{INTRODUÇÃO}

O processo de troca térmica entre dois fluidos em diferentes temperaturas é um fenômeno muito comum em aplicações de engenharia, realizado por trocadores de calor. Do ponto de vista físico e de construção, reconheceu-se que trocadores multitubulares com chicanas permitem um maior coeficiente de troca térmica. No entanto, notou-se experimentalmente que o fluxo do lado do casco está sujeito a interações entre parâmetros fluidodinâmicos e de construção.

As chicanas são usadas para direcionar o fluido do lado do casco através do feixe de tubos e a escolha estrutural na construção é fundamental no projeto do trocador de calor adequado para atender o serviço de forma mais eficiente e econômica. A Figura 1 mostra dois tipos de chicanas, fracionada e helicoidal. 
Figura 1 - Tipos de chicanas: (a) Fracionada. Fonte: Taborek (1983) e (b) Helicoidais. Fonte: Tao et al. (2010).

(a)

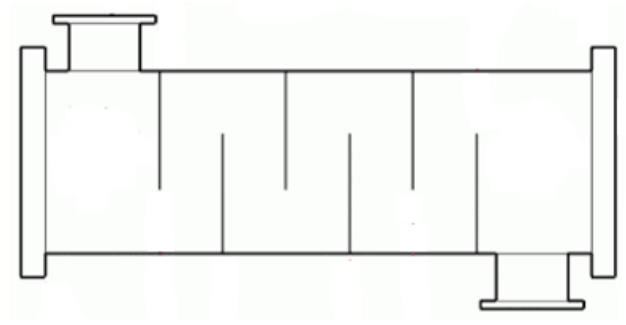

(b)

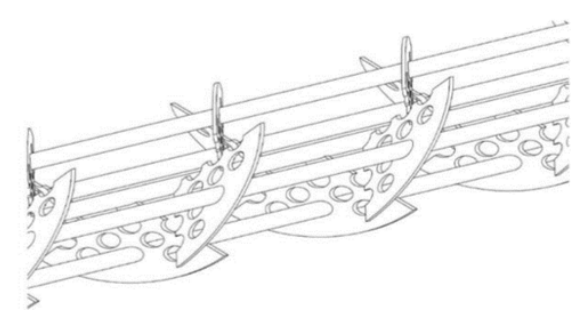

As chicanas fracionadas são empregadas para diminuir a área do escoamento no lado do casco, proporcionando uma maior turbulência, para mudar a direção do escoamento, forçando um fluxo cruzado e para interromper o crescimento da camada limite na parede externa dos tubos, onde ocorre a resistência ao fenômeno de transferência de energia, que acarreta no aumento do coeficiente de película.

Os equipamentos do tipo Helixchanger® são trocadores de calor casco e tubos que possuem uma alteração estrutural nas chicanas, que são instaladas em hélice para que o escoamento seja em forma de parafuso ao longo do comprimento, maximizando o coeficiente de película por reduzir zonas mortas susceptíveis á formação de depósitos. Este tipo de escoamento faz com que a perda de pressão no lado do casco seja menor, sendo geralmente indicado em situações onde a perda de pressão é um fator crítico.

Para comparação do comportamento termofluidodinâmico dos trocadores casco e tubos 1-2 com chicanas fracionadas (CTCF) e chicanas helicoidais $(\mathrm{CTCH})$, faz-se necessário a utilização do modelo diferencial transiente apresentado por Pereira et al. (2015).

$$
\begin{aligned}
& \frac{\partial\left(t_{1}\right)}{\partial t}=\frac{-w \cdot c p_{t} \frac{\partial\left(t_{1}\right)}{\partial x}+U_{1} \cdot N_{1} \cdot P \cdot\left(T-t_{1}\right)}{\rho_{t} \cdot c p_{t} \cdot N_{1} \cdot A_{t}} \\
& \frac{\partial\left(t_{2}\right)}{\partial t}=\frac{w \cdot c p_{t} \frac{\partial\left(t_{2}\right)}{\partial x}+U_{2} \cdot N_{2} \cdot P \cdot\left(T-t_{2}\right)}{\rho_{t} \cdot c p_{t} \cdot N_{2} \cdot A_{t}}
\end{aligned}
$$

$$
\frac{\partial(T)}{\partial t}=\frac{W \cdot C p \cdot \frac{\partial(T)}{\partial x}-U_{1} \cdot N_{1} \cdot P \cdot\left(T-t_{1}\right)-U_{2} \cdot N_{2} \cdot P \cdot\left(T-t_{2}\right)}{\rho_{c} \cdot c p_{c^{\prime}} \cdot A_{c}}
$$

Como o coeficiente de transferência de calor e perda de carga para o casco depende do tipo de chicana, o método de Bell-Delaware (1980) e o método de Tao et al. (2010) foram empregados para estimá-los em CTCF e CTCH, respectivamente.

\subsection{Método Bell-Delaware}


O método de Bell (1980) é o mais preciso para determinar o coeficiente de película e queda de pressão no casco, como constatado em diversos trabalhos na literatura (Taborek, 1983), e baseia-se no modelo proposto por Tinker.

No trabalho de Bell (1980), são definidos os fatores de correção para as correlações de transferência de calor em um escoamento ideal e o modelo para perda de carga no casco. Taborek (1983) traz o detalhamento do método de Bell-Delaware que apresenta a Equação 4 e Equação 5 como o coeficiente de transferência de calor real no casco e perda de carga, respectivamente.

$$
\begin{aligned}
& h_{\text {casco }}=\left(J_{c} \cdot J_{L} \cdot J_{b} \cdot J_{R} \cdot J_{S} \cdot J_{\mu}\right) \cdot h_{\text {ideal }} \\
& \Delta P_{\text {total }}=\Delta P_{c}+\Delta P_{w}+\Delta P_{e}
\end{aligned}
$$

\subsection{Método Tao et al.}

Trocadores do tipo Helixchanger ${ }^{\circledR}$ podem ser dimensionados utilizando um método analítico integral proposto por Tao et al. (2010), onde as correlações do coeficiente convectivo foram baseadas em Schlünder (1983) e Stehlik et al. (1994).

Estes fatores abrangem correções do fluxo helicoidal, do gradiente de temperatura, das variações das propriedades físicas, entre outros. A Equação 6 e seus fatores são encontrados em Stehlik et al.(1994). Os fatores de correção também são utilizados para a perda de pressão, dessa forma a perda de pressão total é expressa pela Equação 7.

$$
\begin{aligned}
& h_{\text {casco }}=\frac{2 . k}{\pi \cdot d_{e}} 0.62\left(0.3+\sqrt{N u_{\text {laminar }}^{2}+N u_{\text {turbulento }}^{2}}\right) Y_{2} \cdot Y_{3} \cdot Y_{4} \cdot Y_{7} \cdot Y_{8} \cdot Y_{9} \cdot Y_{10} \\
& \Delta P_{\text {total }}=\Delta P_{\text {fluxo }}+\Delta P_{\text {ent_saida }}+\Delta P_{\text {bocal }}
\end{aligned}
$$

\section{COMPARAÇÕES DOS MÉTODOS}

Todos os parâmetros relativos à configuração do trocador e as condições operacionais para o Caso 1 e Caso 2 estão apresentados na Tabela 1 e Tabela 2.

A Figura 2 ilustra os perfis de temperatura e coeficiente global ao longo do trocador e observa-se uma pequena diferença nas temperaturas de saída, onde o $\mathrm{CTCH}$ se mostra mais eficiente, possibilidando um comprimento menor para atender as mesmas espeficicações. Nota-se também que o escoamento parafuso maximiza o coeficiente de transferência de calor.

Tabela 1 - Parâmetros do trocador de calor casco e tubos 1-2.

\begin{tabular}{ccccccc}
\hline Casco & $\begin{array}{c}\text { Diâmetro } \\
\text { interno }(\mathrm{m})\end{array}$ & $\begin{array}{c}\text { Diâmetro } \\
\text { externo }(\mathrm{m})\end{array}$ & $\begin{array}{c}\text { Espaçamento das } \\
\text { chicanas }(\mathrm{m})\end{array}$ & $\begin{array}{c}\text { Comprimento } \\
\text { do trocador }(\mathrm{m})\end{array}$ & $\begin{array}{c}\text { Corte da } \\
\text { chicanas }\end{array}$ & $\begin{array}{c}\text { Angulação das } \\
\text { chicanas }\left({ }^{\circ}\right)\end{array}$ \\
\hline 0,387 & 0,400 & 0,304 & 4,900 & 0,25 & 44 \\
\hline Tubos & $\begin{array}{c}\text { Diâmetro } \\
\text { interno }(\mathrm{m})\end{array}$ & $\begin{array}{c}\text { Diâmetro } \\
\text { externo }(\mathrm{m})\end{array}$ & $\begin{array}{c}\text { Pitch dos tubos } \\
(\mathrm{m})\end{array}$ & $\begin{array}{c}\text { Número de } \\
\text { tubos (TEMA) }\end{array}$ & $\begin{array}{c}\text { Tipo de } \\
\text { passo }\end{array}$ & \\
\end{tabular}



0,016
0,019
0,023
160
triângular

Tabela 2 - Condições de operação para os Casos 1 e 2.

\begin{tabular}{ccccccc}
\hline \multicolumn{3}{c}{ Caso 1 } & \multicolumn{3}{c}{ Caso 2 } \\
\hline Fluido & $\begin{array}{c}\text { Temperatura de } \\
\text { entrada (K) }\end{array}$ & Vazão (Kg/s) & Fluido & $\begin{array}{c}\text { Temperatura de } \\
\text { entrada (K) }\end{array}$ & Vazão (Kg/s) \\
\hline Casco & água & 307,0 & 22,0 & benzeno & 400,0 & 20,0 \\
Tubos & água & 297,0 & 35,2 & tolueno & 280,0 & 15,0 \\
\hline
\end{tabular}

Figura 2 - Perfis de temperatura e coef. global para o Caso 1: (a) CTCF e (b) CTCH.

(a)
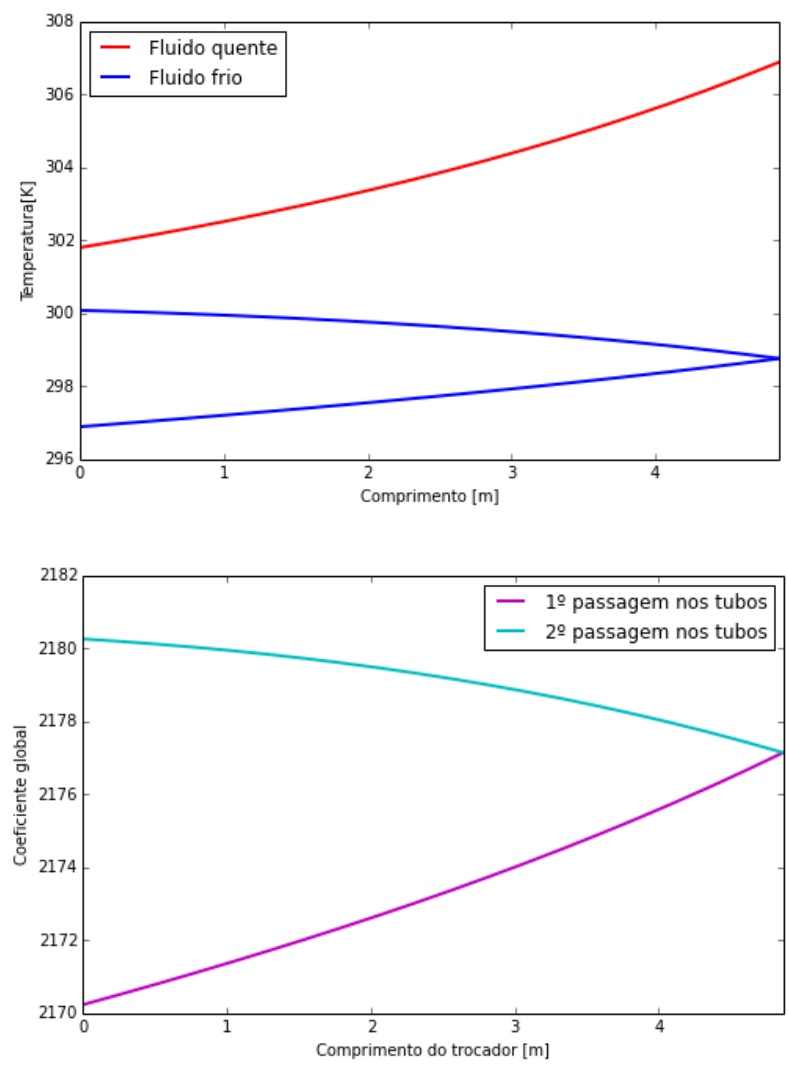

(b)
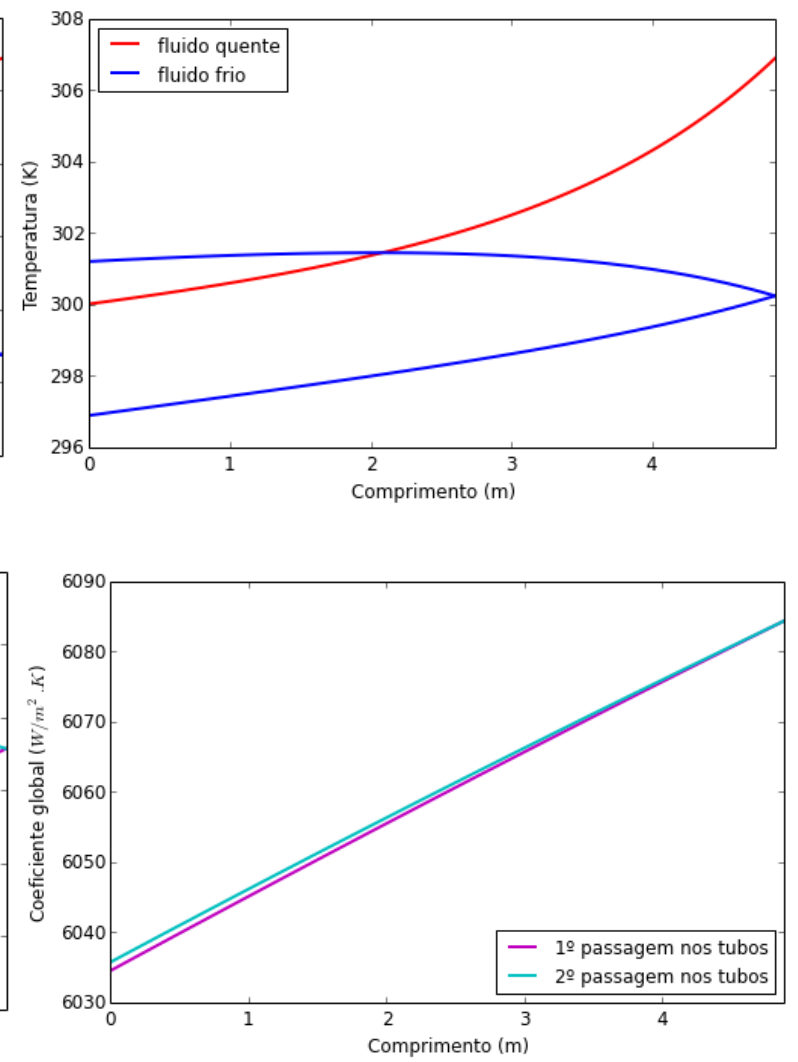

Percebe-se pela Figura 3, que não há vantagem relacionada ao tempo de transiência, já que para os dois tipos o regime estacionário é estabelecido após aproximadamente $25 \mathrm{~s}$.

Para o Caso 2, os perfis de temperatura e coeficiente global são expressivamente diferentes, confirmando que o $\mathrm{CTCH}$ apresenta vantagens térmicas por conta da alteração estrutural das chicanas.

$\mathrm{Na}$ Tabela 3, pode-se verificar que a perda de carga no casco é sempre menor para o $\mathrm{CTCH}$, pois a disposição das chicanas faz com que o escoamente tenha menor resistência. 
Dessa forma, esse tipo de equipamento com chicanas helicoidais é mais indicado por possuir uma carga térmica maior e uma perda de pressão consideravelmente menor.

Figura 3 - Perfis de temperatura com o tempo para o Caso 1: (a) CTCF e (b) CTCH.

(a)

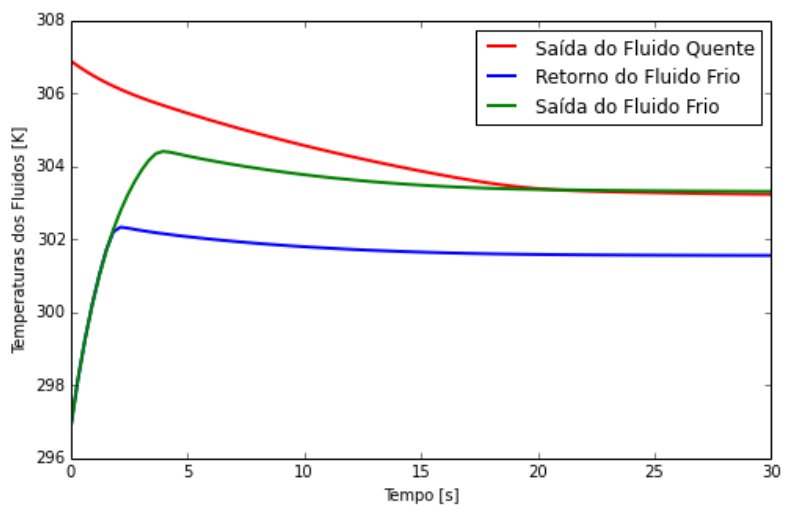

(b)

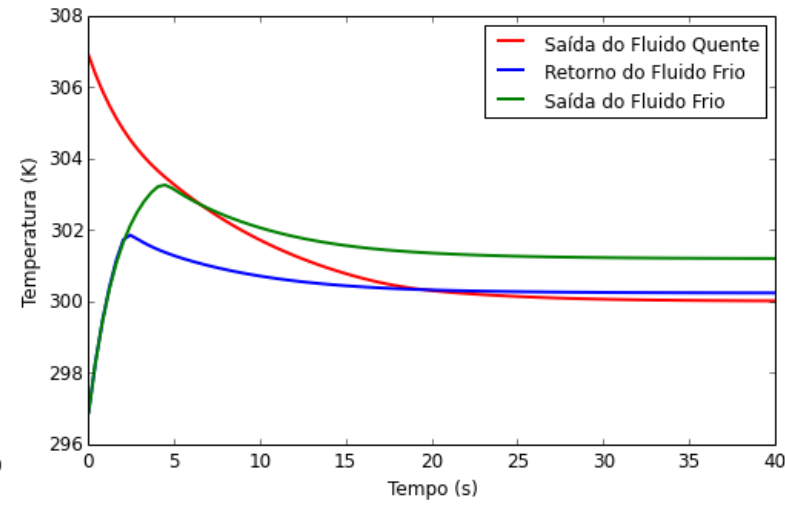

Figura 4 - Perfis de temperatura e coef. global para o Caso 2: (a) CTCF e (b) CTCH.

(a)
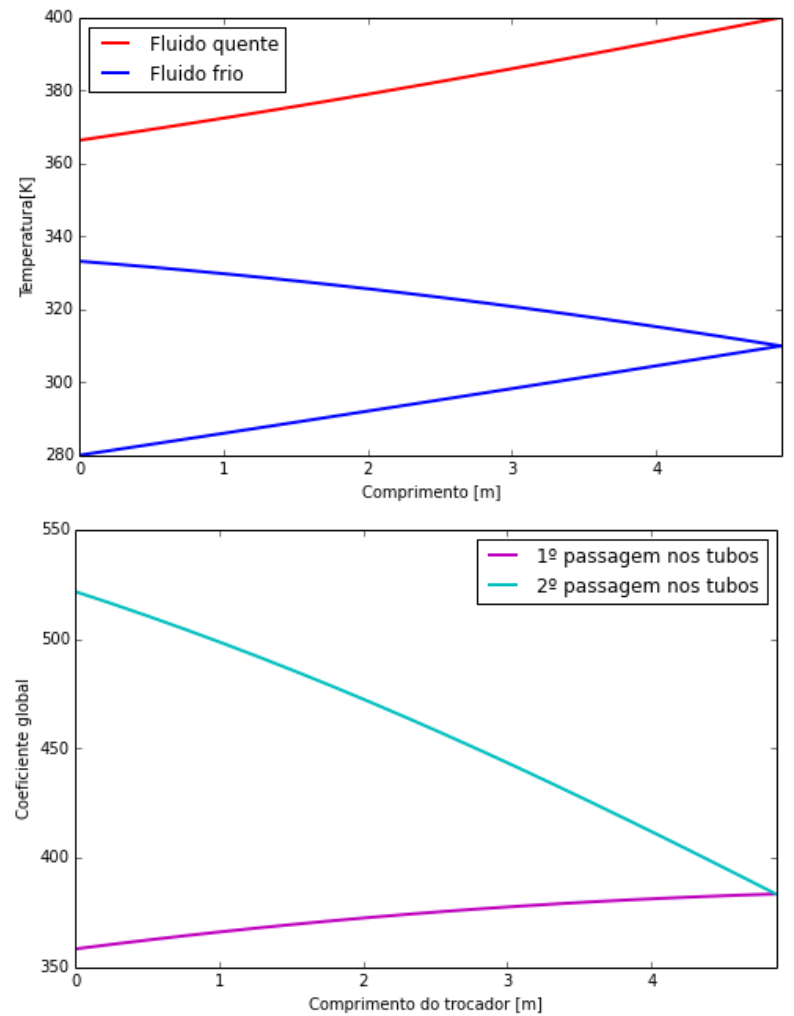

(b)
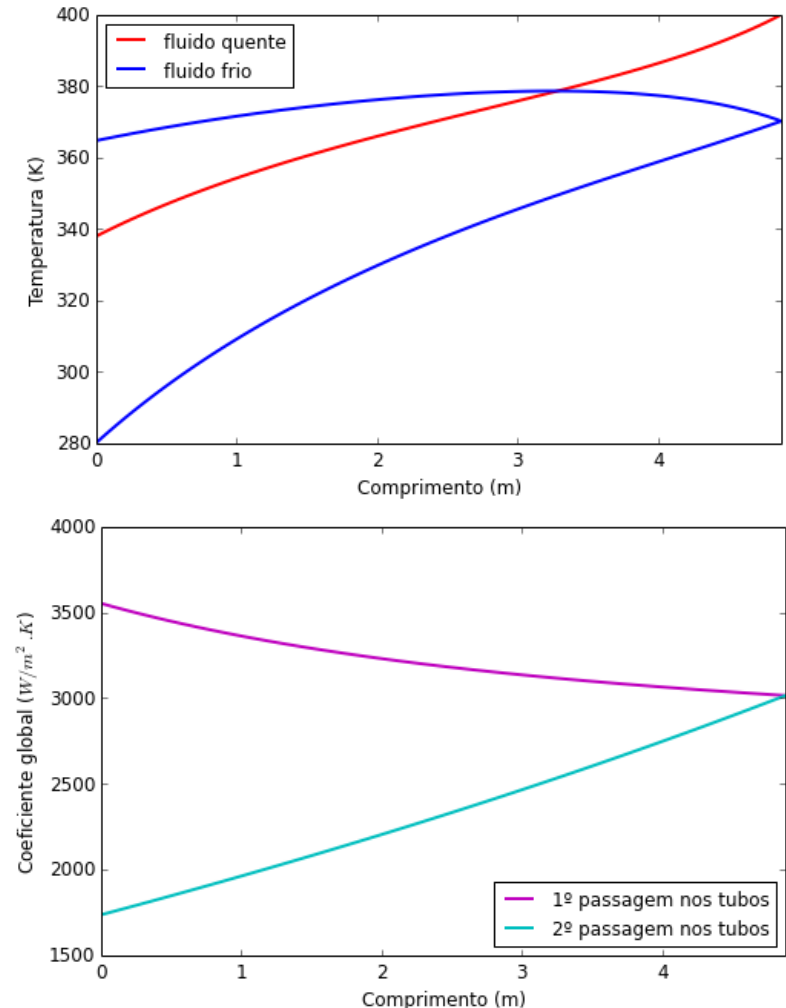

Tabela 3 - Perda de pressão e carga térmica para os Casos 1 e 2.

\begin{tabular}{ccccccc}
\hline & \multicolumn{4}{c}{ Caso 1 } & \multicolumn{3}{c}{ Caso 2 } \\
\hline & $\Delta P_{\text {casco }(\mathrm{KPa})}$ & $\Delta P_{\text {tubos }(\mathrm{KPa})}$ & $\begin{array}{c}\text { Carga } \\
\text { termica }(\mathrm{KW})\end{array}$ & $\Delta P_{\text {casco }(\mathrm{KPa})}$ & $\Delta P_{\text {tubos }}(\mathrm{KPa})$ & $\begin{array}{c}\text { Carga } \\
\text { termica }(\mathrm{KW})\end{array}$ \\
\hline CTCF & 60,272 & 62,661 & 660,848 & 28,565 & 13,18 & 1375,851
\end{tabular}




\begin{tabular}{lrrrrrr}
$\mathrm{CTCH}$ & 55,263 & 55,707 & 717,432 & 26,94 & 21,24 & $4.737,098$ \\
\hline
\end{tabular}

\section{CONCLUSÃO}

Mostrou-se, pelas simulações, que o trocador de calor com chicana helicoidal é mais eficiente do que com chicana fracionada, pois apresenta maior carga térmica e menor perda de pressão menor.

\section{NOMENCLATURA}

$\begin{array}{ll}h_{\text {ideal }} & \text { coeficiente de película para bando de tubos ideal }\left[\mathrm{W} / \mathrm{m}^{2} \mathrm{~K}\right] \\ J & \text { fatores de correção para o método de Bell-Delaware } \\ \Delta P_{c} & \text { perda de pressão pelo fluxo cruzado [Pa] } \\ \Delta P_{w} & \text { perda de pressão pela espaçamento nas janelas [Pa] } \\ \Delta P_{e} & \text { perda de pressão de entrada e saída do trocador [Pa] } \\ t_{1} & \text { temperatura local do fluido frio na primeira passagem no trocador }[\mathrm{K}] \\ t_{2} & \text { temperatura local do fluido frio na segunda passagem no trocador }[\mathrm{K}] \\ T & \text { temperatura do fluido quente }[\mathrm{k}] \\ U_{1} & \text { coeficiente de convecção local na primeira passagem no trocador }\left[\mathrm{W} / \mathrm{m}^{2} . \mathrm{K}\right] \\ U_{2} & \text { coeficiente de convecção local na segunda passagem no trocador }\left[\mathrm{W} / \mathrm{m}^{2} . \mathrm{K}\right] \\ Y_{n} & \text { fatores de correção para o método de Tao et al. } \\ w & \text { vazão mássica do fluido frio }[\mathrm{Kg} / \mathrm{s}] \\ W & \text { vazão mássica do fluido quente }[\mathrm{Kg} / \mathrm{s}]\end{array}$

\section{REFERÊNCIAS}

BELL, K. J., Delaware Method for Shell Side Design, Heat Exchanger Thermal-Hydraulic Fundamentals and Design, New York: McGraw-Hill, 1980.

PEREIRA, A. S.; MAGAlHAES, M. L.; CARTAXO, S. J. M. Development of Transient Differential Model of Bell-Delaware Method with A Case Study of Water $/ \mathrm{TiO}_{2}$ Nanofluid. J. Adv. Thermal Science Research, v. 2, p. 12-21, 2015.

SCHLÜNDER, E. U.; Heat Exchanger Design Handbook. Washington: Hemisphere, 1983.

STEHLIK, P.; NEMCANSKY, J.; KRAL, D.; Comparison of Correction Factors for Shelland-Tube Heat Exchangers with Segmental or Helical Baffles. Heat Transfer Eng., v. 15, p. 55-65, 1994.

TABOREK, "Shell-and-Tube Heat Exchangers", Section 3.3, Heat Exchanger Design Handbook, Hemisphere, 1983. 
TAO, W. Q.; HE, Y. L.; ZHANG, J. F.; A Design and Rating Method for Shell-and-Tube Heat Exchangers with Helical Baffles. Journal of Heat Transfer, v. 132, p. 51802.1 51802.8, 2010. 